\title{
471321 - A TECHNIQUE OF LUNG ISOLATION FOR WHOLE LUNG LAVAGE IN A CHILD WITH PULMONARY ALVEOLAR PROTEINOSIS
}

\author{
Catherine Paquet, MD, Cengiz Karsli, MD, FRCP \\ Department of Anesthesia, Hospital for Sick Children, Toronto, ON, Canada
}

Purpose: To report a case in which lung isolation and whole lung lavage was carried out safely in a small child using two cuffed tracheal tubes without the need for postprocedural ventilation.

Clinical Features: Written parental consent was obtained to present this case. A 2.5year-old, $11 \mathrm{~kg}$ male with lysinuric protein intolerance and pulmonary alveolar proteinosis presented for left lung lavage. In order to secure lung isolation, an airway assembly consisting of two cuffed tracheal tubes (3.0 and $3.5 \mathrm{~mm}$ ID, Portex, USA) and the angled and Y-connectors from a standard double lumen bronchial tube set (Bronchopart, Rusch, Germany) was prepared (Fig). Following anesthetic induction and muscle relaxation the $3.5 \mathrm{~mm}$ ID cuffed tube was inserted in the left main stem bronchus (bronchial tube) and the $3.0 \mathrm{~mm}$ ID tube was placed in the trachea (tracheal tube). Proper tube positioning was verified with a $2.2 \mathrm{~mm}$ OD flexible fiberoptic bronchoscope (Olympus, USA). Lung isolation was also verified clinically by clamping each of the angled connectors while auscultating for breath sounds. A test lavage was performed with 10 cc of normal saline administered via the bronchial tube under bronchoscopic visualization via the tracheal tube to ensure there was no fluid leak. Left lung lavage then proceeded and lavage returns were collected by gravity after each aliquot. Anesthesia was maintained with propofol and remifentanil infusions, titrated to effect. The patient's trachea was extubated at the end of the procedure and his symptoms improved over the next few days. The patient returned to the operating room two days later to have the same procedure performed on the right lung. A similar anesthetic technique was used and bronchial lavage was completed in the same manner. The patient's trachea was extubated at the end of the case and his recovery in the post-anesthetic care unit was uneventful. Conclusion: We report the successful and safe use of two cuffed tracheal tubes in a two year old patient in order to perform whole lung lavage.

References: Thorax 2000 55: 67-77 N Eng J Med 2003 349: 2527-39 Resp Res 2005 6: 138 


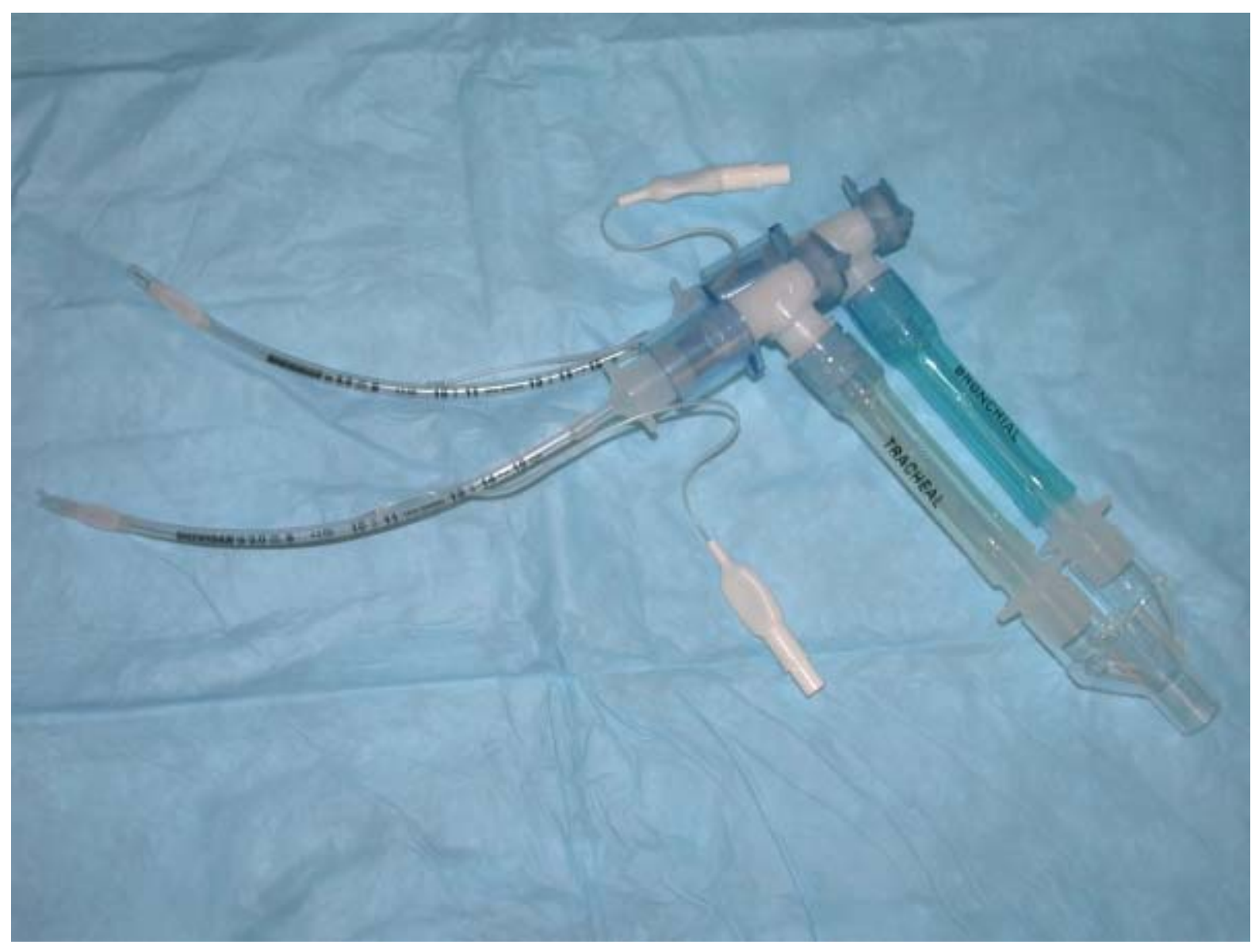

